\title{
Gender differences in cardiac left ventricular mass and function: Clinical and experimental observations
}

\author{
Eduardo Manuel Escudero, Alejandro Orlowski, Alejandro Díaz, \\ Oscar Andrés Pinilla, Irene Lucía Ennis, Ernesto Alejandro Aiello \\ Centro de Investigaciones Cardiovasculares, Facultad de Ciencias Médicas, \\ Universidad Nacional de La Plata, CONICET, La Plata, Argentina
}

\begin{abstract}
Background: The aim of this study was to evaluate gender-associated impact on left ventricular mass (LVM) and on left ventricular function (LVF) in humans and rats with aging.

Methods: Myocyte area and collagen volume fraction (CVF) were studied in rats. LVM and LVF were evaluated in animals and humans by echocardiography and LVM index (LVMI) was obtained.
\end{abstract}

Results: LVMI, myocyte area and CVF were similar in males and females of 1-month-old rats. LVMI in children was similar in both genders. In contrast, in 6-month-old rats (5 males and 5 females), LVMI (17.7 $\pm 0.7 \mathrm{mg} / \mathrm{mm}$ vs. $10.1 \pm 0.2 \mathrm{mg} / \mathrm{mm} ; p<0.01)$, and myocyte area $\left(4572.5 \pm 72.6 \mu m^{2}\right.$ vs. $\left.3293.85 \pm 57.8 \mu m^{2}, p<0.01\right)$ were higher in male animals without differences in CVF. Men $(n=25)$ exhibited greater LVMI than women $(n=25)\left(77.4 \pm 3.2 \mathrm{~g} / \mathrm{m}^{2}\right.$ vs. $\left.63.3 \pm 1.8 \mathrm{~g} / \mathrm{m}^{2}, p<0.01\right)$, whereas the LVF was higher in women $(105.9 \pm 2.9 \%$ vs. $95.3 \pm 3.5 \%, p<0.01)$.

Conclusions: There is a clear gender-associated impact on LVM with aging in humans and rats. Similar CVF and LVF associated to greater myocyte size and LVM in male rats suggest a process of physiological response. However, the increase in cardiac mass without an associated improved cardiac function in men in comparison to women could likely represent a potential disadvantage in the adaptive response during growth. (Cardiol J 2014; 21, 1: 53-59)

Key words: sex differences, cardiac myocytes, cardiac hypertrophy, cardiac function

\section{Introduction}

It is well known that during early natal period cardiomyocytes normally stop increasing in number (hyperplasia of the fetal period) and that the further increase in heart size is due to cellular hypertrophy [1]. However, despite the fact that the number of cardiac myocytes is genetically determined and reaches a final amount within the first year after birth [2], the possibility of cardiac self-renewal has been re-examined over the years [3-5].

Although myocytes constitute approximately $30-35 \%$ of the total myocardial ventricular cells, they represent approximately $60 \%$ of the total myocardial volume [6]; hence, the increase in ventricular mass is mainly a consequence of the increase in the size or number of myocytes.

Address for correspondence: Dr Eduardo Manuel Escudero, $\mathrm{PhD}, \mathrm{MD}$, Centro de Investigaciones Cardiovasculares, Facultad de Ciencias Médicas, UNLP-CONICET, Calle 60 y 120, 1900, La Plata, Argentina, e-mail: emescu@gmail.com

Received: 12.03.2013 Accepted: 23.07.2013 
Even though left ventricular mass (LVM) is larger in adult men than in adult women [7] — similar to what has been reported in adult rats [8] - this difference was not detected when female and male children or weaning female and male rats $[9,10]$ were studied.

This divergence in the left ventricular (LV) weight in adult humans and/or rats might be due to sex differences in myocardial mass (and hence the number of myocytes) that was programmed in early life and/or different cardiac growth with certain level of myocyte hypertrophy during development in males.

In addition to the differences in LVM referred, several authors also found different ventricular function according to the sex in studies accomplished in humans and rats $[11,12]$. That difference in left ventricular function (LVF) linked to the increase of LVM could indicate the development of an adaptive hypertrophy according to the sex.

On this basis, the current clinical and experimental observations performed in humans and rats tested the hypothesis that the aging process may have distinct impact on the LVF and structure, being able to develop non-adaptative mechanisms depending on gender.

\section{Methods}

\section{Experimental observations}

Experiments were conducted in accordance with the Guide for the care and use of laboratory animals (NIH publication № 85-23, revised 1966) with normotensive rats of both sexes from the Wistar strains rats. Ten 1-month-old (5 males) and ten 6 -month-old ( 5 males) rats were used in order to have pre-puberal and adult animal samples. All animals were fed ad libitum and had free access to tap drinking water.

Arterial systolic blood pressure (BP) was weekly determined by the tail-cuff method in each animal. Rats were lightly anesthetized $(35 \mathrm{mg} / \mathrm{kg}$ pentobarbital sodium, IP) to perform 2-dimensional targeted M-mode tracings according to established recommendations [13]. Measurements were made using criteria analogous to those recommended by the American Society of Echocardiography for using in humans in 3 consecutive cardiac cycles. All tracings were analyzed with an off-line analysis system (Image pro Plus 6.0). LVM was calculated according to Devereaux and Reichek [14], and LVM index (LVMI) was obtained normalizing LVM to tibial length. Relative diastolic wall thickness was calculated as posterior wall thickness/LV end-diastolic radius [15].
LV systolic function was evaluated by corrected endocardial fractional shortening expressed as percentage of the expected value, on the basis of a regression equation between afterload, assessed by calculating circumferential end-systolic stress, and endocardial fractional shortening [16].

Animals were euthanized under anesthesia and their hearts were removed to weigh the LV. $\mathrm{LV}$ weight was divided by tibial length to determine the LVMI. In 2 rats out of each group ventricular tissue was fixed in buffered $10 \%$ formaldehyde and paraffin embedded to stain with picrosirius red for determining collagen volume fraction as previously described [17]. Perivascular collagen was excluded from this measurement.

In the other rats of each group, cardiac myocytes were isolated as previously described [18] in order to measure the cell apparent surface area via the detection of the edges with the Image Pro Plus software. The area of 200 myocytes per rat heart was measured.

\section{Clinical observations}

The studied population consisted of 96 normotensive subjects, 6 to 24 years of age, without antecedents of cardiovascular diseases, obesity and with normal echocardiograms. This cohort was divided into 2 groups: 46 pre-adolescents, Tanner stage III [19], 6 to 12 years old ( 21 girls, 25 boys); and 50 young of 18 to 24 years of age ( 25 women, 25 men).

Three consecutive measurements of arterial systolic and diastolic BP in 2 office visits were taken, according to the Seventh Report of the Joint National Committee on Prevention, Detection, Evaluation and Treatment of High Blood Pressure [20] and the Fourth Report of the Joint National Committee on Diagnosis, Evaluation, and Treatment of High Blood Pressure in children and adolescents [21]. The averages of the systolic and diastolic BP measurements were used in the analysis.

The weight and height data were obtained in each included subject in order to calculate the body surface area (BSA) [22]; an echocardiogram was performed following the recommendations of the American Society of Echocardiography [23], using commercially available equipment provided with $2-4 \mathrm{MHz}$ or $5-7 \mathrm{MHz}$ phased-array transducers. LVMI was calculated by normalizing LVM to BSA. Other parameters employed to evaluate the LV structure and function were obtained as explained above for the experimental observations. 
Table 1. Blood pressure, anthropometic and echocardiographic parameters in 1-month-old rats.

\begin{tabular}{lccc}
\hline & Males & Females & P \\
\hline $\mathrm{N}$ & 5 & 5 & \\
Systolic blood pressure [mm Hg] & $107.88 \pm 1.56$ & $108.33 \pm 0.92$ & $\mathrm{NS}$ \\
Weight [g] & $88.60 \pm 3.12$ & $87.60 \pm 3.19$ & $\mathrm{NS}$ \\
Tibial length [mm] & $21.99 \pm 0.22$ & $21.91 \pm 0.10$ & $\mathrm{NS}$ \\
Left ventricular end-diastolic diameter [mm] & $3.75 \pm 0.05$ & $3.70 \pm 0.05$ & $\mathrm{NS}$ \\
Left ventricular end-systolic diameter [mm] & $1.82 \pm 0.04$ & $1.77 \pm 0.05$ & $\mathrm{NS}$ \\
Left ventricular diastolic wall thickness [mm] & $1.03 \pm 0.03$ & $1.00 \pm 0.01$ & $\mathrm{NS}$ \\
Corrected fractional endocardial shortening [\%] & $99.08 \pm 0.69$ & $100.93 \pm 1.43$ & $\mathrm{NS}$ \\
Relative wall thickness & $0.55 \pm 0.02$ & $0.55 \pm 0.03$ & $\mathrm{NS}$ \\
\hline
\end{tabular}

\section{Statistical analysis}

Data are reported as mean \pm standard error. The 2-tailed t-test for independent samples was used for comparing differences between genders in continuous variables. 2-way ANOVA test followed by ad-hoc correction were used for multiple comparisons (males vs. females within the same age range, but also young vs. adult within the same gender). The statistical analyses of the different variables were performed with the Statistical Package for Social Science (SPSS, Chicago, Ill) version 15.0. Statistical significance was assumed for 2 -tailed $\mathrm{p}<0.05$.

\section{Results}

\section{Experimental observations}

Arterial systolic BP, anthropometric and echocardiographic parameters of 1-month-old rats are presented in Table 1. Body and heart weights, tibial length and BP were not significantly different between male and female animals. LV diastolic diameter and wall thickness were similar in these male and female rats, as shown in Table 1 . Non-significant difference was found in LV chamber function between male and female rats at this age. LVMI and the surface area of the myocytes were similar in both sexes, as shown in Figure 1. In addition, interstitial collagen volume was similar in male and female rats, as shown in Figure 2.

When the analysis was performed in 6-month-old rats (Table 2), we found that male rats had higher body weight and tibial length. It is interesting to note that $\mathrm{BP}$ was higher in these rats (male and female) than in rats of 1 month of age.

In 6-month-old rats $\mathrm{LV}$ diameter and wall thickness were higher in males than in females but LV geometry and LV systolic function were similar between the genders. In the same way LV

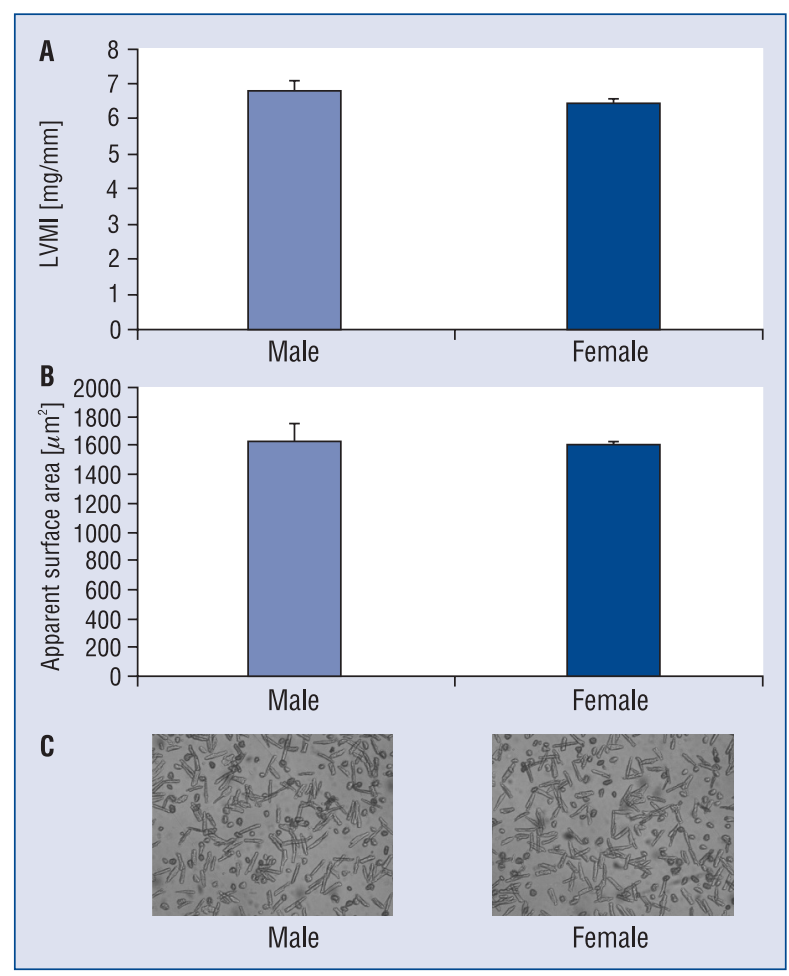

Figure 1. Heart weight and myocyte size in 1-month-old rats. A. Bar graphs showing left ventricular mass index (LVMI) obtained by echocardiogram from male and female rats; B. Apparent surface area of myocytes obtained from male and female rats; $\mathbf{C}$. Representative photographs of isolated myocytes obtained from male and female rats.

systolic function was similar between the rats of 1 and 6 months of age despite the sex (Tables 1,2).

Myocyte size and LVMI were higher in male than female rats at this age (Fig. 3); however, collagen volume and LV systolic function were similar in both genders (Fig. 4). This finding allows us to speculate that the increase in the myocardial cells 


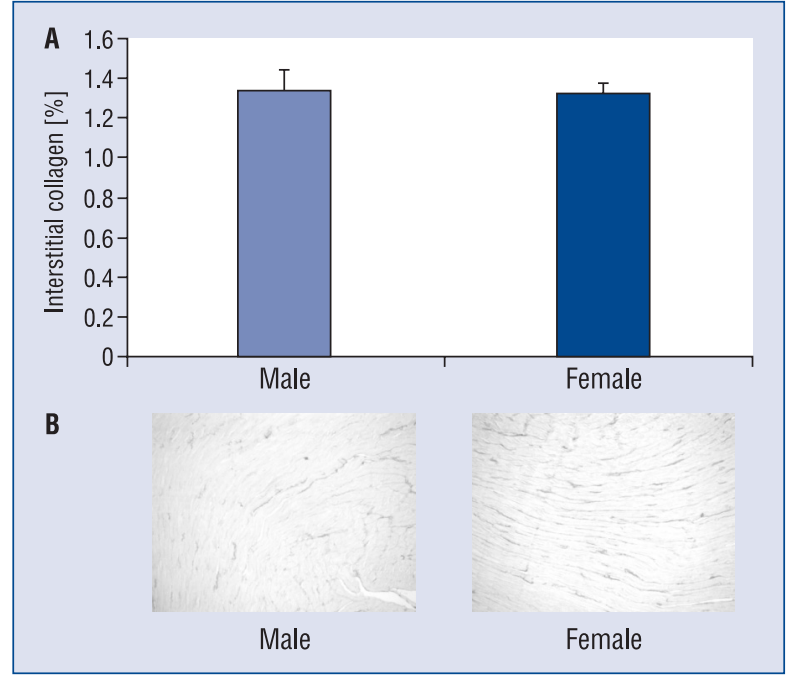

Figure 2. Left ventricular myocardial interstitial collagen in 1-month-old rats. A. Bar graphs showing left ventricular interstitial collagen in male and female rats; B. Histological sample of left ventricular stained with picrosirius red to see interstitial collagen in a representative male and female rat.

size without associated interstitial fibrosis, at least up to this age, may correspond with a physiological adaptation to growth despite the higher BP.

\section{Clinical observations}

Mean age, BSA, arterial BP, and echocardiographic structural and functional parameters of boys and girls were similar (Table 3 ). However, in young population we found differences between sexes, since the weight, height, BSA and arterial $\mathrm{BP}$ were higher in men than in women (Table 4). Structural parameters of LV diameter and wall thickness were also higher in men (Table 4). Relative wall thickness was similar in men and women. Young men presented approximately $44 \mathrm{~g}$ more

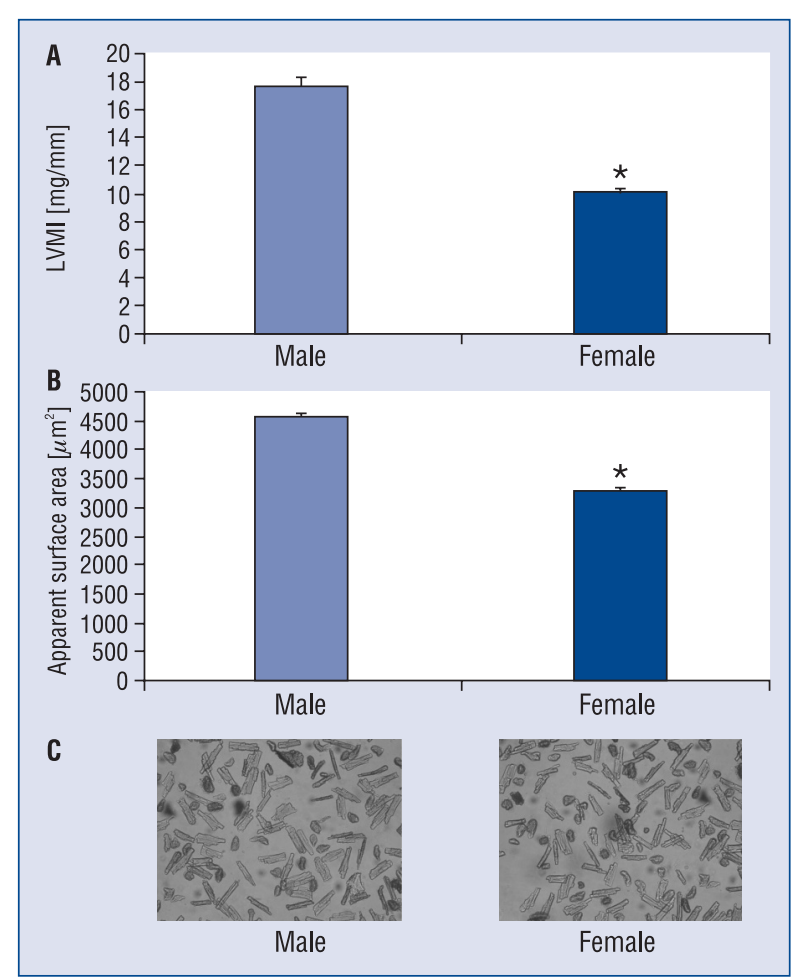

Figure 3. Heart weight and myocyte size in 6-month-old rats. A. Bar graphs showing left ventricular mass index (LVMI) obtained by echocardiogram from male and female rats; B. Apparent surface area of myocytes obtained from male and female rats; $\mathbf{C}$. Representative photographs of isolated myocytes obtained from male and female rats; ${ }^{*} p<0.01$.

LVM than young women in correspondence with higher LVMI, as shown in Figure 5. It is interesting to point out that LV systolic function indexes were higher in woman than in men, as observed in the same figure (Fig. 5).

The analysis of LV systolic function indicates that women have better function than girls (girls:

Table 2. Blood pressure, anthropometic and echocardiographic parameters in 6-months-old rats.

\begin{tabular}{lccc}
\hline & Males & Females & P \\
\hline $\mathrm{N}$ & 5 & 5 & $\mathrm{NS}$ \\
Systolic blood pressure [mm Hg] & $118.00 \pm 3.00$ & $116.40 \pm 1.57$ & $<0.01$ \\
Weight [g] & $402.55 \pm 15.62$ & $214.64 \pm 2.23$ & $<0.01$ \\
Tibial length [mm] & $40.92 \pm 1.35$ & $35.54 \pm 0.64$ & $<0.01$ \\
Left ventricular end-diastolic diameter [mm] & $6.78 \pm 0.20$ & $5.48 \pm 0.10$ & $<0.05$ \\
Left ventricular end-systolic diameter [mm] & $2.55 \pm 0.16$ & $2.10 \pm 0.01$ & $<0.01$ \\
Left ventricular diastolic wall thickness [mm] & $1.62 \pm 0.01$ & $1.26 \pm 0.01$ & $\mathrm{NS}$ \\
Corrected fractional endocardial shortening [\%] & $100.61 \pm 2.01$ & $99.37 \pm 1.58$ & $\mathrm{NS}$ \\
Relative wall thickness & $0.48 \pm 0.02$ & $0.46 \pm 0.01$ & \\
\hline
\end{tabular}




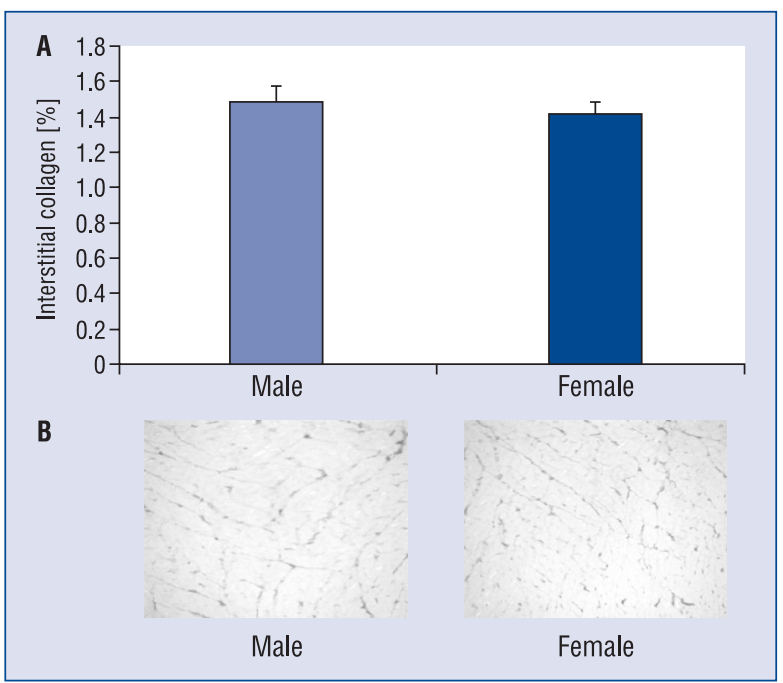

Figure 4. Left ventricular myocardial interstitial collagen in 6-month-old rats; A. Bar graphs showing left ventricular interstitial collagen in male and female rats; B. Histological sample of left ventricular stained with picrosirius red to see interstitial collagen in a representative male and female rat.

$96.71 \pm 3.23 \%$; women: $105.94 \pm 2.92 \%$; $<<0.02$ ), whereas no significant differences were observed between young boys and adult men (boys: $94.67 \pm$ $\pm 3.64 \%$; men: $95.35 \pm 3.50 \%$; NS).

\section{Discussion}

Since cardiomyocytes represent the largest percentage of heart volume $(\approx 60 \%)$ and, as a consequence, have the major impact on determining LVM, similar LVM in different population might mean similar cardiomyocyte number and/or size. Our results showing similar myocyte size in 1-month-old rats allow us to confirm that assumption. Considering that the number of cardiac myocytes is genetically determined and might reach a final amount early in development, we could speculate that the final number of cells could be different depending on gender. However, since we observed the same size in cardiomyocytes and LVM in female and male young rats, this possibility could be dismissed.

The results of our clinical observations in children are in agreement with our experimental observations, showing similar LVM in boys and girls, allowing us to extend the previous considerations.

In coincidence to other authors [24-28], we found that LVM in adult rats and human beings was higher in males than in females. Consistently, Campbell et al. [27] also showed that all measured cellular dimensions of cardiomyocytes isolated from adult rats were significantly smaller in females compared to males. In addition, we did not find differences in the collagen percentage between sexes. This last evidence would allow us to discard the assumption that the higher LVM in these animals could represent a phenotype of pathological hypertrophy. The different size of the myocytes that explain the differences found in LVM between genders can be the consequence of different responses to similar stimuli in male cells or to dissimilar overload signals depending on sex (i.e. arterial BP, BSA, stroke work or the presence of specific hormones with different effect in myocyte growth).

Another factor worth-mentioning is the possibility that not only the size but also the number of

Table 3. Blood pressure, anthropometric and echocardiographic parameters in children.

\begin{tabular}{lccc}
\hline & Boys & Girls & P \\
\hline $\mathrm{N}$ & 25 & 21 & \\
Weight $[\mathrm{kg}]$ & $34.82 \pm 2.01$ & $30.33 \pm 2.79$ & $\mathrm{NS}$ \\
Height $[\mathrm{cm}]$ & $133.44 \pm 2.49$ & $128.24 \pm 3.86$ & $\mathrm{NS}$ \\
Body surface area $\left[\mathrm{m}^{2}\right]$ & $1.12 \pm 0.04$ & $1.03 \pm 0.06$ & $\mathrm{NS}$ \\
Systolic blood pressure [mm Hg] & $108.64 \pm 1.88$ & $107.53 \pm 2.01$ & $\mathrm{NS}$ \\
Diastolic blood pressure [mm Hg] & $72.44 \pm 1.99$ & $72.10 \pm 1.93$ & $\mathrm{NS}$ \\
Mean blood pressure [mm Hg] & $84.51 \pm 1.90$ & $83.91 \pm 1.82$ & $\mathrm{NS}$ \\
Left ventricular end-diastolic diameter [mm] & $39.37 \pm 0.79$ & $37.46 \pm 0.93$ & $\mathrm{NS}$ \\
Left ventricular end-systolic diameter [mm] & $22.89 \pm 0.80$ & $22.00 \pm 0.86$ & $\mathrm{NS}$ \\
Interventricular septum end-diastolic diameter [mm] & $5.89 \pm 0.14$ & $6.13 \pm 0.23$ & $\mathrm{NS}$ \\
Left ventricular posterior wall end-diastolic thickness [mm] & $6.35 \pm 0.22$ & $6.31 \pm 0.27$ & $\mathrm{NS}$ \\
Relative wall thickness & $0.31 \pm 0.01$ & $0.33 \pm 0.01$ & $\mathrm{NS}$ \\
Corrected fractional endocardial shortening [\%] & $96.71 \pm 3.23$ & $94.67 \pm 3.64$ & $\mathrm{NS}$ \\
\hline
\end{tabular}


Table 4. Blood pressure, anthropometic and echocardiographic parameters in young people.

\begin{tabular}{lccc}
\hline & Men & Women & P \\
\hline $\mathrm{N}$ & 25 & 25 \\
Weight $[\mathrm{kg}]$ & $72.04 \pm 1.86$ & $55.64 \pm 1.29$ & $<0.01$ \\
Height $[\mathrm{cm}]$ & $175.64 \pm 1.30$ & $161.08 \pm 1.27$ & $<0.01$ \\
Body surface area $\left[\mathrm{m}^{2}\right]$ & $1.87 \pm 0.03$ & $1.58 \pm 0.02$ & $<0.01$ \\
Systolic blood pressure $[\mathrm{mm} \mathrm{Hg}]$ & $125.86 \pm 2.02$ & $115.30 \pm 1.96$ & $<0.01$ \\
Diastolic blood pressure $[\mathrm{mm} \mathrm{Hg}]$ & $77.38 \pm 1.58$ & $72.63 \pm 1.51$ & $<0.02$ \\
Mean blood pressure [mm Hg] & $93.08 \pm 1.43$ & $86.85 \pm 1.53$ & $<0.01$ \\
Left ventricular end-diastolic diameter [mm] & $47.70 \pm 0.84$ & $42.36 \pm 0.65$ & $<0.01$ \\
Left ventricular end-systolic diameter [mm] & $28.67 \pm 0.89$ & $23.49 \pm 0.57$ & $<0.01$ \\
Interventricular septum end-diastolic diameter [mm] & $8.53 \pm 0.24$ & $7.67 \pm 0.19$ & $<0.01$ \\
Left ventricular posterior wall end-diastolic thickness $[\mathrm{mm}]$ & $9.33 \pm 0.22$ & $8.09 \pm 0.19$ & $<0.01$ \\
Relative wall thickness & $0.38 \pm 0.01$ & $0.37 \pm 0.01$ & $\mathrm{NS}$ \\
\hline
\end{tabular}

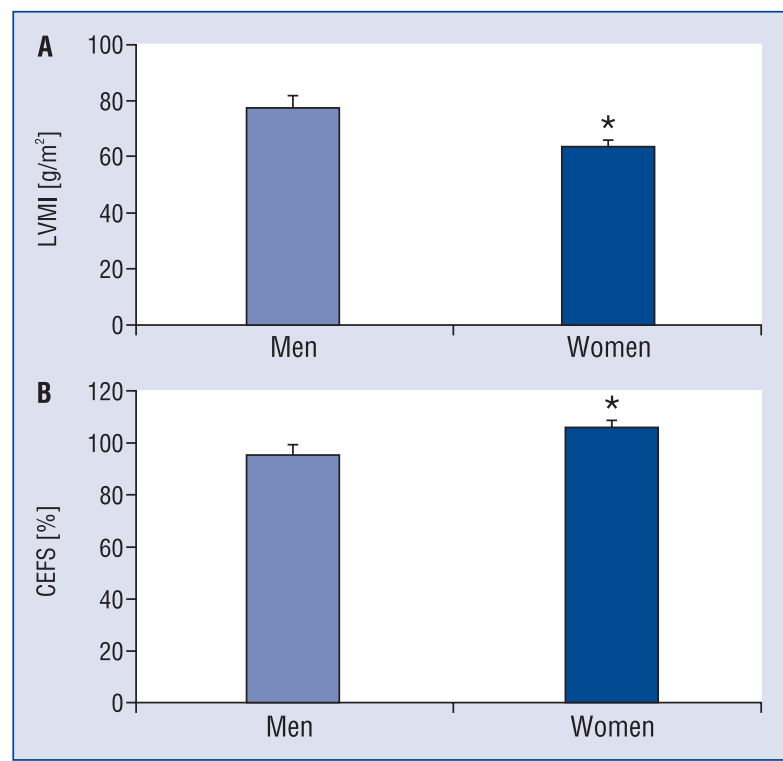

Figure 5. Left ventricular mass index and function in men and women; A. Bar graphs showing the differences in left ventricular mass index (LVMI) between men and women; B. Bar graphs showing the differences in left ventricular systolic function between women and men; CEFS - corrected endocardial fractional shortening; ${ }^{*} \mathrm{p}<0.01$.

cardiomyocytes could be differently contributing to the increase of LVM in males in comparison to females. On the other hand, it should be considered that the smaller size of the myocytes found in older female rats could be associated to greater apoptosis, thus contributing to explain the minor LVM detected. However, Olivetti et al. [28] studying the size and number of cardiomyocytes in 53 women and 53 men observed a larger reduction in the number of the cells in men, with the increment of the volume associated to an enlargement of LVM according to the increasing age. These pieces of evidence suggest that the interrelation between size and number of myocytes during life time would explain the differences in LVM according to gender.

Our results obtained in human beings are in agreement with investigations that have documented differences in systolic LVF according to gender in favor of women [29, 30]. Several mechanisms were considered to explain this phenomenon, as enhancement of calcium influx into cardiomyocyte secondary to the increase in the density of sarcolemmal calcium channels in women [29] or augmentation of the activity of the actomyosin ATP-ase with a rise in calcium sensitivity of contractile proteins, depending on estrogen levels [30]. On the other hand, the results of our experimental observations, which are in agreement with the study of Weinberg et al. [31], demonstrate that LVF was similar in rats of both genders. These data suggest that in contrast to humans estrogen levels might not have impact on the LVF of rats.

\section{Limitations of the study}

Considering that the relation between the number and size of cardiomyocytes would allow us to explain the difference in LVM between genders, the main limitation of the study was the inability to obtain the number of cells in the experimental observations. This limitation was based on the difficulty of having a reliable methodology to accomplish that task. On the other hand, in clinical observations, the impossibility to obtain myocardial biopsies obliged us to extrapolate to the experimental findings to interpret the results. 


\section{Conclusions}

In summary, the main results of this study evidence an obvious age influence on the LVM according to sex, both in rats and humans. Young rats and children show similar LVM in both sexes, whereas in older population LVM is greater in males than in females. The lack of development of fibrosis with increase in the size of myocytes and LVM in male rats, as well as the lack of alteration in LV systolic function, would suggest a process of adaptation or physiological response. However, although normal healthy adult men display a LVF that fully satisfies the perfusion demands of the body, the increase in cardiac mass without an associated improved cardiac function in men in comparison to women could likely represent a potential disadvantage in the adaptive response during growth. Thus, it is feasible to speculate that this phenomenon could partly explain the higher prevalence of cardiovascular disease in men than in pre-menopausic women. Further studies are needed to bring new insights into this matter.

\section{Conflict of interest: none declared}

\section{References}

1. Zac R. Development and proliferative capacity of cardiac muscle cells. Circ Res, 1974; 35: 17-26

2. Anversa P, Leri A, Kajstura J, Nadal-Ginard B. Myocyte growth and cardiac repair. J Mol Cell Cardiol, 2002; 34: 91-105.

3. Beltrani AP, Urbanek K, Kajstura J, Yan SM, Finato N. Evidence that human cardiac myocytes divide after myocardial infarction. N Engl J Med, 2001; 344: 1750-1757.

4. Nadal-Ginard B, Kajstura J, Leri A, Anversa P. Myocyte death, growth and regeneration in cardiac hypertrophy and failure. Circ Res, 2003; 92: 139-150.

5. Bastos Carvalho A, Campos de Carvalho AC. Heart regeneration: Past, present and future. World J Cardiol, 2010; 2: 107-111.

6. Nag AC. Study of non-muscle cells of the adult mammalian heart: A fine structural analysis and distribution. Cytobios, 1980; 28: $41-61$.

7. Salton CJ, Chuang ML, O’Donnell CJ et al. Gender differences and normal left ventricular anatomy in an adult population free of hypertension: A cardiovascular magnetic resonance study of the Framingham Heart Study Offspring cohort. J Am Coll Cardiol, 2002; 39: 1055-1060.

8. Weinberg EO, Thienelt CD, Katz SE et al. Gender differences in molecular remodeling in pressure overload hypertrophy. J Am Coll Cardiol, 1999; 34: 264-273.

9. Szewczykowska M, Grygalewicz J, Szkiłładź-Skiba M. Left ventricular mass in children in relation to body surface area. Med Wieku Rozwoj, 2009; 13: 187-193.

10. Parente LB, Aguila MB, Mandarim-de-Lacerda CA. Deleterious effects of high-fat diet on perinatal and postweaning periods in adult rat offspring. Clin Nutr, 2008; 27: 623-633.

11. Harrison WK, Smith JE. Sex differences in cardiac function of a group of young adults. Cardiology, 1980; 66: 74-84.

12. Schaible TF, Scheuer J. Comparison of heart function in male and female rats. Basic Res Cardiol, 1984; 79: 402-412.
13. Jones EF, Harrap SB, Calafiore P, Tonkin AM. Development and validation of echocardiographic methods for estimating left ventricular mass in rats. Clin Experimental Pharmacol Physiology, 1992; 19: 361-364.

14. Devereux RB, Reichek N. Echocardiographic determinations of left ventricular mass in man: anatomic validation of the method. Circulation, 1977; 55: 613-618.

15. Ganau A, Devereux RB, Roman MJ et al. Patterns of left ventricular hypertrophy and geometric remodelling in essential hypertension. J Am Coll Cardiol, 1992; 19: 1550-1558.

16. de Simone G, Devereux RB, Roman MJ et al. Assessment of left ventricular function by the midwall fractional shortening-end-systolic stress relation in human hypertension. J Am Coll Cardiol, 1994; 23: 1444-1451.

17. Pérez NG, Piaggio MR, Ennis IL et al. Phosphodiesterase 5A inhibition induces $\mathrm{Na}+\mathrm{H}+$ exchanger blockade and protection against myocardial infarction. Hypertension, 2007; 49: 1095-1103.

18. Aiello EA, Villa-Abrille MC, Escudero EM et al. The myocardial hypertrophy of the WKY rats. Am J Physiol Heart Circ Physiol, 2004; 286: 1229-1235.

19. Tanner JM. The measurement of maturity. Trans Eur Orthod Soc, 1975: 45-60

20. Chobanian AV, Bakris GL, Black HR et al. Joint National Committee on Prevention, Detection, Evaluation, and Treatment of High Blood Pressure. National Heart, Lung, and Blood Institute; National High Blood Pressure Education Program Coordinating Committee. Seventh report of the Joint National Committee on Prevention, Detection, Evaluation, and Treatment of High Blood Pressure. Hypertension, 2003; 42: 1206-1252.

21 . The fourth report on the diagnosis, evaluation, and treatment of high blood pressure in children and adolescents. Pediatrics, 2004; 114: 555-576.

22. DuBois D, DuBois EF. A formula to estimate the approximate surface area if height and weight be known. Arch Intern Med, 1916; 17: 863-871.

23. Lang RM, Bierig M, Devereux RB et al. American Society of Echocardiography's Nomenclature and Standards Committee; Task Force on Chamber Quantification; American College of Cardiology Echocardiography Committee; American Heart Association; European Association of Echocardiography, European Society of Cardiology. Recommendations for chamber quantification. J Am Soc Echocardiogr, 2005; 18: 1440-1463.

24. de Simone G, Devereux RB, Daniels SR, Meyer RA. Gender differences in left ventricular growth. Hypertension, 1995; 26 : 979-983.

25. Douglas PS, Katz SE, Weinberg EO, Chen MH, Bishop SP, Lorell HL. Hypertrophic remodeling: gender differences in the early response to left ventricular pressure overload. J Am Coll Cardiol, 1998; 32: 1118-1125.

26. Bai SL, Campbell SE, Moore JA, Morales MC, Gerdes AM. Influence of age, growth, and sex on cardiac myocyte size and number in rats. Anat Rec, 1990; 226: 207-212.

27. Campbell SE, Rakusan K, Gerdes AM. Change in cardiac myocyte size distribution in aortic-constricted neonatal rats. Basic Res Cardiol, 1989; 84: 247-258.

28. Olivetti G, Giordano G, Corradi D et al. Gender differences and aging: effects on the human heart. J Am Coll Cardiol, 1995; 26: 1068-1079.

29. Buonanno C, Arbustini E, Rossi L et al. Left ventricular function in men and women. Another difference between sexes. Eur Heart J, 1982; 3: 525-528.

30. Hayward CS, Kelly RP, Collins P. The roles of gender, the menopause and hormone replacement on cardiovascular function. Cardiovasc Res, 2000; 46: 28-49.

31. Weinberg EO, Thienelt CD, Katz SE et al. Gender differences in molecular remodeling in pressure overload hypertrophy. J Am Coll Cardiol, 1999; 34: 264-273. 